\title{
ENFERMEDAD PLUS EN LA RETINOPATÍA DEL PREMATURO DE GESTACIÓN MÚLTIPLE. ANÁLISIS DE RIESGO
}

\section{RETINOPATHY OF PREMATURITY IN MULTIPLE BIRTHS: RISK ANALYSIS FOR PLUS DISEASE}

\author{
GARCÍA-SERRANO JL ${ }^{1}$, RAMÍREZ-GARCÍA MC², PIÑAR-MOLINA R ${ }^{3}$
}

\begin{abstract}
RESUMEN
Objetivo: Determinar los factores de riesgo asociados con la enfermedad plus en la retinopatía del prematuro (ROP).

Método: En los últimos 8,5 años, realizamos un estudio prospectivo sobre la ROP en gemelos y tri1lizos. Cincuenticuatro prematuros nacidos de parto múltiple con peso $(\leq 1500$ gr) y edad gestacional ( $\leq 32$ semanas).

Resultados: Los factores que presentaron asociado un mayor riesgo de enfermedad plus fueron: grado avanzado de ROP, mayor área sin vascularizar, menor edad gestacional, menor peso al nacer, existencia de ductus arteriovenoso persistente, mayor número de días intubado, mayor número de eventos adversos, menor puntaje en el test Apgar 2 y una menor ganancia de peso postnatal. Con regresión logística binaria, sólo el grado más avanzado de ROP (OR: 5,$5 ; \mathrm{p}<0,009)$ y la menor ganancia de peso posnatal (OR: 0,$58 ; \mathrm{p}<0,04)$ resultaron factores predictivos del desarrollo de la enfermedad plus. En las 4-6 primeras semanas de vida, los niños con enfermedad plus obtenían una pobre ganancia media de peso de 3,9 $\pm 3,1$ gr/día frente a los 11,84 $\pm 8,3 \mathrm{gr} /$ día que alcanzaban aquellos $\sin$ enfermedad plus $(\mathrm{p}<0,0001)$.
\end{abstract}

\section{ABSTRACT}

Purpose: To analyze the risk factors associated with plus disease in retinopathy of prematurity (ROP).

Method: Over a period of 8.5 years we carried out a prospective study of ROP in twins and triplets. Fifty-four multiple-birth infants with low birth weight $(\leq 1500 \mathrm{~g})$ and low gestational age $(32 \leq$ weeks) were admitted to the University Hospital of Granada.

Results: Logistic regression analyses showed the following variables to be associated with an increased risk of plus disease: severe ROP, large area of avascular retina, low gestational age, low birth weight, a patent ductus arteriosus, length of mechanical ventilation, adverse events increase, low $5 \mathrm{~min}$ Apgar scores and poor postnatal weight gain (in the first 4 to 6 weeks of life).

Using multiple logistic regression, only the grade of ROP (OR: $5.5 ; \mathrm{p}<0.009$ ) and poor postnatal weight gain (OR: $0.58 ; \mathrm{p}<0.04)$ were predictive factors of development of plus disease. Infants with «plus» disease gained an average $3.9 \pm 3.1 \mathrm{~g} /$ day in the first 6 weeks of life, compared to a mean of $11.84 \pm 8.3$ $\mathrm{g} /$ day for those without plus disease $(\mathrm{p}<0.0001)$. Conclusion: Advanced ROP stages and poor weight gain were the most significant factors asso-

Recibido: 31/5/08. Aceptado: 13/4/09.

Servicio de Oftalmología. Unidad de Gestión Clínica. UCIP Pediátrica y Neonatal. Hospital Universitario San Cecilio de Granada. Granada. España.

1 Doctor en Medicina. Facultativo Especialista de Área de Oftalmología.

2 Diplomada en Enfermería. Supervisora. Unidad de Cuidados Intensivos Pediatría y Neonatal.

3 Diplomada en Enfermedad. Unidad de Cuidados Intensivos Pediatría y Neonatal. Responsable del Programa ROP. 
Conclusiones: Los factores más significativamente asociados con la enfermedad plus eran el estadio avanzado de ROP y la pobre ganancia de peso. Los gemelos que ganan más de 7 gr/día de peso, en las primeras 4-6 semanas de vida, significativamente reducen el riesgo de enfermedad plus. Una buena ganancia de peso postnatal es una estrategia efectiva contra la ceguera evitable de la ROP.

Palabras clave: Retinopatía del prematuro, enfermedad plus, factores de riesgo, ganancia de peso, parto multiple, efectos adversos, puntaje Agpar. ciated with plus disease. Twins who gained weight at more than $7 \mathrm{~g} /$ day in the first 4-6 weeks of life had a significantly reduced risk of plus disease. A good weight gain is an effective strategy against avoidable blindness due to ROP (Arch Soc Esp Oftalmol 2009; 84: 191-198).

Key words: Retinopathy of prematurity. plus disease, risk factors, weight gain, multiple-birth, adverse effects, Apgar score.

\section{INTRODUCCIÓN}

En la retinopatía del prematuro (ROP) los mayores predictores de riesgo son: el estadio avanzado de la ROP, un área más extensa de retina sin vascularizar, una mayor velocidad de progresión de la ROP (1) y la existencia de enfermedad plus. Si a la ROP severa se asocia la enfermedad plus, el riesgo de encontrar un resultado estructural desfavorable se incrementa en 8,6 veces (1-3).

La enfermedad plus se caracteriza por tortuosidad arterial y dilatación venosa del polo posterior y se acepta como un estadio igual o superior a una imagen fotográfica estándar definida por el grupo ETROP (4).

Para el diagnóstico de enfermedad plus se requería que estas anormalidades vasculares ocuparan al menos dos cuadrantes (5). Y en un intento de adelantar el diagnóstico de esta enfermedad se definió a la enfermedad pre-plus como aquella en la que existían cambios vasculares mayores de lo normal, sin llegar al estadio plus (6-8).

En nuestra muestra de niños nacidos de parto múltiple, incluidos en el protocolo de la retinopatía del prematuro, nuestro objetivo fue determinar que factores de riesgo modificables permiten reducir el riesgo de enfermedad plus.

\section{SUJETOS, MATERIAL Y MÉTODOS}

El presente trabajo representa a los niños nacidos de parto múltiple, incluidos en el protocolo de la retinopatía del prematuro del Hospital Universitario San Cecilio de Granada, entre los años 2000 a 2008 (8,5 años). Recoge un total de 54 niños prematuros, 6 niños nacidos de dos gestaciones triples y los 48 restantes nacidos de parto gemelar. La UCI de Pediatría del Hospital asiste a una población media de 423.000 habitantes, de las áreas de Granada y Motril.

Los prematuros nacidos de parto múltiple entran en el protocolo de la retinopatía del prematuro, en mayor porcentaje, que los nacidos de parto simple. Su elevada morbilidad y la fecundación in vitro con más de dos embriones implantados, son factores que contribuyeron a este hecho diferencial (9).

Al estudiar el grado de la retinopatía del prematuro, se consideró que alcanzaron la enfermedad plus aquellos que tenían un grado de tortuosidad y dilatación igual o superior a la fotografía estándar del ETROP (4). Para este trabajo sólo se consideraron aquellos que llegaron a la enfermedad plus e ignoramos aquellos que presentando enfermedad pre-plus no avanzaron hasta enfermedad plus.

La lente de 20 dioptrías produce una magnificación de 2,5, parecida a la encontrada en la fotografía standard y abarca un área de retina de unos $40^{\circ}$, lo que representa unos ocho diámetros papilares. La lente de 28 dioptrías produce una magnificación de 1,5 y abarca un área de retina de $60^{\circ}$, lo que representa unos doce diámetros papilares (10).

Se utilizó la lente de 28 dioptrías para valorar la extensión de las lesiones y el grado de retinopatía del prematuro (6), y la lente de 20 dioptrías para valorar la enfermedad plus. Con la lente de 20 dioptrías se midió, en diámetros papilares, el área de retina temporal sin vascularizar.

Si con la lente de 20 dioptrías se visualiza la periferia de la retina, y la vascularización desde el polo posterior sólo llega hasta la mitad de la lente, restaría por vascularizar el equivalente a cuatro diámetros papilares. Si la vascularización ocupa 3/4 de la lente equivaldría a dos diámetros papilares sin vas- 
cularizar. También equivale a dos diámetros papilares cuando indentamos periféricamente y los vasos quedan justo delante del área indentada de retina.

Se ha realizado un estudio de cohortes, y se ha analizado a la enfermedad plus como factor dependiente y como factores independientes a aquellos que influyen en la aparición de la ROP. Las variables independientes analizadas fueron las siguientes: grado ROP, diámetros de retina temporal sin vascularizar, edad gestacional semanas, presencia de ductus, días de administración de oxígeno (intubación, CPAP, gafas), peso al nacer, grado de membrana hialina, test de Apgar, efectos adversos, circunstancias maternas y del embarazo (edad de la madre, diabetes gestacional, hipertensión materna, adicción a drogas, cesárea, parto univitelino o bivitelino), comorbilidad (hemorragia periintraventricular, sepsis, patología ECO o TAC, cirugía de drenaje para hidrocefalia), fármacos o sangre administrada (surfactante, teofilina, transfusión sanguínea).

Se define como efectos adversos o comorbilidad a todas aquellas maniobras médicas o quirúrgicas que no hallamos en un parto vaginal normal y sin complicaciones postoperatorias. También se considera así a la patología encontrada en el niño prematuro que no hallamos en un niño sano y maduro al nacer.

Se realiza un análisis de regresión logística binaria de la enfermedad plus en relación a cada una de las variables predictivas y un análisis de regresión múltiple logística con el programa XLSTAT 2008.2.02 (Addinsoft Inc. New York, USA). Se ha considerado a cada gemelo como un niño independendiente.

\section{RESULTADOS}

Entre los años 2000-2008 fueron explorados dentro del programa de la retinopatía del prematuro 292 niños, de ellos 54 niños nacieron de parto múltiple $(18,49 \%)$. La incidencia de parto múltiple en nuestra área es de uno de cada 85 partos. La proba- bilidad de que un niño de parto múltiple sea incluido en el protocolo de la ROP es significativamente mayor que la de un niño nacido de parto simple (tabla I).

En nuestra serie de 54 niños de parto múltiple, presentan enfermedad plus doce niños, el 22\% (12/54). De estos doce niños, siete recibieron ablación retiniana el (58\%) (siete de doce).

No se encontró asociación estadísticamente significativa en la enfermedad plus (variable dependiente) y las siguientes variables independientes:

- Días de administración de Oxigeno en CPAP o con gafas.

- Realización de parto por cesárea.

- Realización de transfusiones sanguíneas.

- Puntaje de sufrimiento fetal al nacimiento: Apgar 1.

- Grado de hemorragia periintraventricular $(\mathrm{p}<$ 0,09, n.s.).

- Sepsis.

- Administración de indometacina, teofilina o surfactante.

- En relación con la madre: edad, diabetes gestacional, adicción a drogas, e hipertensión materna.

- Realización de cirugía de derivación por hidrocefalia (11).

- Respecto a los gemelos: evolución del gemelo mejor y peor, y nacimiento univitelino o bivitelino.

Las variables significativas con el análisis univariante quedan expuestas en la tabla II.

El grado de retinopatía del prematuro explica un $74,9 \%$ de la probabilidad de presentar enfermedad plus. Aquellos pacientes que en la primera exploración de fondo de ojo, a las 4-6 primeras semanas de vida, presentaban un mayor área temporal sin vascularizar tenían un riesgo significativamente más elevado de presentar enfermedad plus.

Se encontró que el riesgo de presentar enfermedad plus en el niño era del $4,77 \%$ y si la nacida era niña del 33,44\%. Sin embargo con 900 gramos o menos de peso al nacer se encontraron un total de doce prematuros (nueve niñas y tres niños). De

Tabla I. ODDS del niño de parto múltiple/parto simple sobre la posibilidad de entrar en el protocolo de ROP (9,53); de recibir ablación retiniana por su $\mathrm{ROP}(2,07)$. De presentar un resultado estructural desfavorable $(1,16)$

\begin{tabular}{|c|c|c|c|c|c|c|c|}
\hline \multirow{2}{*}{$\begin{array}{l}\text { Años 2000-2008 } \\
\text { Número niños }\end{array}$} & \multicolumn{2}{|c|}{$\begin{array}{l}\text { Parto simple } \\
\text { Esperado/real }\end{array}$} & \multicolumn{2}{|c|}{$\begin{array}{c}\text { Múltiple } \\
\text { Esperado/real }\end{array}$} & \multicolumn{2}{|c|}{ ODDS $(95 \%)$} & \multirow{2}{*}{$\begin{array}{c}\text { Significación } \\
\mathrm{P}<0,0001\end{array}$} \\
\hline & 285,28 & 238 & 6,79 & 54 & 9,53 & $(4,2-21,6)$ & \\
\hline Crio/láser reti. & & 16 & & 7 & 2,07 & $(0,72-5,7)$ & No significati. \\
\hline Res. Est. Desf. & (32 ojos) & 4 & (14 ojos) & 2 & 1,16 & $(0,43-3,1)$ & No significati. \\
\hline
\end{tabular}


Tabla II. Factores clínicos que predicen el riesgo de enfermedad plus en el análisis univariante. Odds ratio y correlación ajustada entre cada factor y enfermedad plus

\begin{tabular}{lccc}
\hline Factor clínico & Odds ratio $(95 \%$ CI) & Valor p & $\mathrm{R}^{2}$ Nagelkerke \\
\hline Estadio más avanzado ROP & $33,36(3,45-322)$ & $\mathrm{p}<0,00001$ & $74,9 \%$ \\
Ganancia peso adicional (g/día) 4-6 sem. & $0,75(0,62-0,91)$ & $\mathrm{p}<0,002$ & $36,5 \%$ \\
Edad gestacional (semana adicional) & $0,52(0,35-0,78)$ & $\mathrm{p}<0,001$ & $33,9 \%$ \\
Máxima área temporal sin vascularizar & $1,78(1,23-2,6)$ & $\mathrm{p}<0,002$ & $29,3 \%$ \\
Peso al nacer (cada 100 g más) & $0,66(0,49-0,88)$ & $\mathrm{p}<0,005$ & $28,4 \%$ \\
Ductus (presencia vs ausencia) & $13,0(2,5-66,4)$ & $\mathrm{p}<0,005$ & $26,8 \%$ \\
Días de intubación (cada día adicional) & $1,09(1,02-1,16)$ & $\mathrm{p}<0,005$ & $26,8 \%$ \\
Test Apgar 2 (cada punto adicional) & $0,83(0,71-0,97)$ & $\mathrm{p}<0,02$ & $15,1 \%$ \\
Efectos adversos (efecto adicional) & $1,29(1,02-1,62)$ & $\mathrm{p}<0,032$ & $12,6 \%$ \\
\hline \hline
\end{tabular}

ellos, el $67 \%$ presentaban enfermedad plus.

En las tablas III y IV se desglosan en la columna de la izquierda las variables independientes significativas. En la columna derecha se expresa el porcentaje de riesgo de hallar enfermedad plus en algunos tramos de estas variables.

A mayor puntuación en el test de Apgar en el quinto minuto menor riesgo de enfermedad plus (tabla IV). A mayor edad gestacional existe un puntuación en el test de Apgar 2 significativamente mayor $\left(r^{2}=25 \%, p<0,0002\right)$.

A mayor ganancia de peso en gramos/día en las

Tabla III. Riesgo de enfermedad plus en función de los valores de distintas variables

\begin{tabular}{lc}
\hline Estadio ROP & Riesgo enfermedad plus \\
Estadio 2: & $10,7 \%$ \\
Estadio 3: & $78,9 \%$ \\
Ganancia peso gramos/día: & \\
(4-6 primeras semanas vida) & \\
0 gramos/día & $67 \%$ \\
3 gramos/día & $47 \%$ \\
6 gramos/día & $27 \%$ \\
12 gramos/día & $6,7 \%$ \\
18 gramos/día & $1,35 \%$ \\
Edad gestacional (semanas): & \\
24 semanas & $96 \%$ \\
26 semanas & $83 \%$ \\
28 semanas & 50 \\
30 semanas & $17 \%$ \\
32 semanas & $4 \%$ \\
Área temporal avascular a la primera exploración medido en \\
diámetros papilares \\
8 DP \\
6 DP \\
4 DP \\
2 DP \\
Peso al nacer & $89 \%$ \\
600 gramos & $68 \%$ \\
800 gramos & $33 \%$ \\
1.000 gramos & $11 \%$ \\
1.200 gramos & \\
1.500 gramos & $75 \%$ \\
\hline \hline
\end{tabular}

4-6 primeras semanas, menor riesgo de presentar enfermedad plus (tablas II, V). Si logramos que todos los prematuros ganen $\geq 7$ gramos/día en las 46 primeras semanas, la reducción del riesgo relativo de manifestar enfermedad plus sería del 86\% (RRR). Y el número de pacientes que necesitan ser tratados, elevando la ganancia de peso a $\geq 7$ gramos/día, para lograr que un paciente no presente enfermedad plus será de 2,43 (NNT).

Al realizar el análisis regresión múltiple logística las dos variables que aportan mayor significación en relación a la enfermedad plus son: El grado de retinopatía y la ganancia de peso en las primeras 46 semanas. $\mathrm{r}^{2}=88,3 \%, \mathrm{p}<0,0001$. Con una especificidad del $97,8 \%$, una sensibilidad del $91,7 \%$, y un porcentaje de clasificaciones correctas del 96,6\% El modelo predictivo presenta una razón de verosimilitud, $\mathrm{LR}+>10$ y una $\mathrm{LR}-=0,085$ lo que indica un excelente modelo predictivo.

Las dos únicas variables significativas en el

Tabla IV. Riesgo de enfermedad plus en función del valor de las variables independientes

\begin{tabular}{lc}
\hline Ductus arteriovenoso & $\%$ riesgo enfermedad pluspersistente \\
\hline Sí & $75 \%$ \\
No & $15 \%$ \\
Días de intubación & \\
$\quad$ 0 días & $9 \%$ \\
21 días & $40 \%$ \\
42 días & $81 \%$ \\
Efectos adversos (número) & \\
0 efectos & $4 \%$ \\
4 efectos & $10 \%$ \\
8 efectos & $23 \%$ \\
12 efectos & 44,65 \\
Test de Apgar 2 (número) & \\
Puntuación 0 & $42 \%$ \\
Puntuación 2 & $34 \%$ \\
Puntuación 6 & $20 \%$ \\
Puntuación 10 & $11 \%$ \\
\hline \hline
\end{tabular}


Tabla V. Se observa que los prematuros que obtienen ganancias de peso $<7$ gramos/día, tienen un riesgo significativamente mayor de presentar enfermedad plus que los que ganan $\geq 7$ gramos/día $\left(X^{2}=10,53, p<0,002\right.$, su OR: 14,09 con IC al $95 \%$ de 2,3 a 69,6 )

\begin{tabular}{lcc}
\hline $\begin{array}{l}\text { Ganancia peso 4-6 } \\
\text { primeras semanas }\end{array}$ & $\begin{array}{c}\text { Grado RP } \\
\text { de } 0 \text { a } 3\end{array}$ & Grado 2- 3 plus \\
\hline$\geq 7$ gramos/día & 31 & 2 \\
0-6,99 gramos/día & 11 & 10 \\
$\mathrm{n}=54$ & 42 & 12 \\
\hline \hline
\end{tabular}

modelo binario multivariante eran el grado de ROP, con una odds ratio de 3,945 (IC al 95\% de 2,9-932, $\mathrm{p}<0,009)$ y la ganancia de peso, con una odds ratio de 0,399 (IC al 95\% de 0,46-0,97, p < 0,04).

Para valorar el riesgo de presentar enfermedad plus el grado ROP se estratifica en cuatro niveles (rop3 $=$ pliegue falciforme, rop $2=$ cresta, rop $1=$ línea, rop $0=$ no retinopatía). El peso en gramos $(\mathrm{p}=$ gramos de ganancia/día). El riesgo se calcula con la siguiente formula:

$$
\text { Logit : }-8,04+3,94(\text { grado ROP) }-0,399(p)
$$

$$
\text { Riesgo enfermedad plus }=\frac{1}{1+\mathrm{e}^{-\operatorname{logit}}}
$$

a) En ningún paciente con ROP final grado 0 ó 1

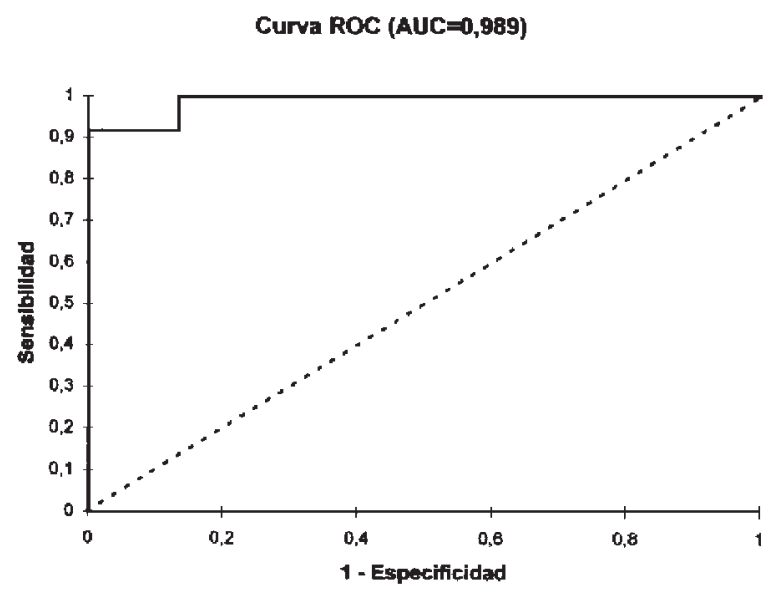

Fig. 1: Curva ROC del modelo predictivo de presentar enfermedad plus. Obtenida con dos variables: grado máximo de ROP y la ganancia de peso/día (4-6 primeras semanas de vida). Área bajo la curva 0,98 IC al $95 \%$ de 0,97 a 1 . encontrábamos enfermedad plus. La ganancia de peso no modificaba el resultado.

b) Retinopatía del prematuro grado 2: Aquellos que no ganaban peso presentaban un riesgo de enfermedad plus del 46,29\%. En aquellos que ganaban 6 gramos/día el riesgo era del 7,31\%, y en los que ganaban 12 gramos /día el riesgo era del 0,71\%.

c) Retinopatía del prematuro grado 3: Aquellos que no ganaban peso presentaban un riesgo de enfermedad plus del 97,8\%; los que ganaban 6 gramos/día, del $80,23 \%$, los que ganaban 12 gramos/día, del 27,03\%; y en aquellos que ganaban 20 gramos/día el riesgo era del $1,5 \%$.

\section{DISCUSIÓN}

La gestación múltiple determina cuatro veces mayor mortalidad perinatal que la gestación simple. También implica mayor riesgo de prematuridad, prolapso del cordón, anoxia en el parto del segundo gemelo, restricción del crecimiento, anomalías estructurales $(12,13)$ y mayor riesgo de ROP severa $(14,15)$. Otros autores no encuentran mayor riesgo de ROP en niños nacidos de parto múltiple $(16,17)$.

Los dos criterios mayores de gravedad de la ROP son el estadio más avanzado y la localización en zona posterior I ó II (4). Tres de cada cuatro pacientes que alcanzaban el estadio 3 de RP necesitaban tratamiento (18). En nuestra serie tres de cada cinco pacientes que alcanzaban estadio plus necesitaban tratamiento.

Los gemelos de igual edad gestacional no reciben la misma nutrición y el mismo flujo vascular intrauterino (18). Esto determina una desigual vascularización retiniana que encontramos en el $44,5 \%$ de nuestros gemelos ya a la primera exploración. Uno de los gemelos suele presentar un área de retina mayor sin vascularizar, habitualmente el niño de menor peso $(87,5 \%)$. Todo ello determina que ya a las 4-6 semanas de vida posnatal existan factores modificables maternos y fetales que pueden influir en el área no vascularizada de la retina (18).

En nuestro trabajo cada semana adicional de madurez intrauterina reduce en un 53\% el riego de llegar hasta la enfermedad plus. Lo mismo ocurre con el peso al nacer, por cada 100 gramos adicionales se reduce el riesgo de enfermedad plus en un $34 \%$. Una reducción de riesgo equivalente describen los autores para la ROP severa $(1,11)$.

La presencia del ductus arteriovenoso determina 
una alta mortalidad y morbilidad asociadas a la enfermedad pulmonar crónica. Los pacientes con ductus arteriovenoso persistente presentan un riesgo de enfermedad plus y de ROP significativamente mayor que los pacientes que no lo presentan $(20,21)$.

La intubación prolongada incrementa el riesgo de retinopatía del prematuro (21), y una intubación de más de 28 días se correlaciona con la aparición de un estadio de ROP de grado 3 o superior (22). Cada día adicional que el niño permanece intubado se incrementa el riesgo global de presentar enfermedad plus en un 1,5\%. Las medidas que ayuden a rebajar el tiempo de intubación (22) pueden reducir el porcentaje de niños con ROP y enfermedad plus susceptibles de tratamiento ablativo (23).

El riesgo de presentar ROP se incrementa cuando los niños prematuros son de muy bajo peso (24), son más inmaduros (25) y están más enfermos (20, 26, 27). En los prematuros con más comorbilidad se incrementa el riesgo de pasar de ROP moderada a ROP severa (28). Nosotros obtenemos que a mayor número de efectos adversos se observa un mayor riesgo de enfermedad plus.

En la mayoría de las series el sexo es neutral o los niños presentan un mayor riesgo de presentar ROP (16). En nuestra serie obtenemos también que el sexo es neutral, y el mayor riesgo de presentar enfermedad plus, encontrado en las niñas, responde a que las niñas de bajo peso suelen sobrevivir en mayor proporción en gestaciones complejas $(10,29)$.

En relación con la puntuación en el test de Apgar en el primer minuto, existe un mayor riesgo de ROP en los que presentan un valor inferior a 6-7 $(23,30,31)$. Hallamos una asociación significativa entre el bajo puntaje del test Apgar 2 y el riesgo de la enfermedad plus. Sin embargo la baja puntuación en el test de Apgar 2 va muy correlacionada con la baja edad gestacional (17), hecho que corroboramos porque a cada semana adicional en su edad gestacional le corresponde el incremento de un punto en el Apgar 2.

En nuestra serie hallamos tres factores modificables asociados a un mayor riesgo de presentar enfermedad plus tres factores: la pobre ganancia de peso postnatal, el elevado número de días intubado y el mayor número de efectos adversos.

Una adecuada nutrición contribuye al crecimiento y desarrollo pulmonar, proporciona protección contra las infecciones y mejora la tolerancia a la toxicidad al oxigeno (32).

Si el niño sigue creciendo y no recibe los nutrien- tes necesarios redistribuye primando a áreas que regulan automatismos fisiológicos como corazón y pulmón y restringe el flujo a otras áreas del sistema nervioso (33).

Los bajos niveles de leptina encontrados en niños prematuros, de menos de 32 semanas, son similares a los encontrados en adultos malnutridos y anoréxicos $(34,35)$. Los bajos niveles de IGF-1 en suero indican malnutrición fetal durante el embarazo humano (36-39). Una infranutrición proteica determina unos bajos niveles de IGF-1 en suero y eleva el riesgo de ROP (40-42). La IGF-1 es un factor protector y predictor del desarrollo de ROP (43). Cuando los niveles del IGF-1 y VEGF ascienden comienza de nuevo el desarrollo vascular en la ROP (38). Por ello, mejorar la nutrición y restaurar los niveles de IGF-1 a aquellos encontrados en el útero a través de la nutrición, o conseguir valores en suero similares a los prematuros sin retinopatía puede ayudar a prevenir la ROP $(43,44)$. Al nacimiento, el peso del prematuro y valor de la IGF-1 presentan una fuerte correlación (39). En las semanas siguientes se observa que la ganancia de peso posnatal se correlaciona positivamente con las concentraciones de IGF-1 $(40,41)$.

Bien por insuficiencia de nutrientes, bien por el daño celular ocasionado por la hipoxia pueden lesionarse los pericitos y las células musculares de los vasos sanguíneos lo que determina una incapacidad para regular el flujo sanguíneo, que contribuye a la vasodilatación y tortuosidad características de la enfermedad plus (45).

Los niños con baja ganancia de peso posnatal presentan mayor riesgo de padecer una retinopatía del prematuro severa (estadio 3 o superior) (46-48). A igual edad gestacional, los prematuros con bajo peso presentan mayor riesgo de ROP y más grave que los niños con peso adecuado (49). La ganancia media de peso fetal intrauterino en gemelos de 27 a 36 semanas es de 18 a 22 gramos/kg/día (50). En nuestra serie, los niños sin enfermedad plus ganan de media 12 gramos/día, y con enfermedad plus esta ganancia se reduce a 4 gramos/día. Por cada gramo adicional de ganancia en su peso posnatal, en las 4-6 primeras semanas de vida, la probabilidad de enfermedad plus es un $25 \%$ menor. Obtener una adecuada ganancia de peso, en la ROP grado 2 , anula el riesgo de presentar un resultado estructural desfavorable. En la ROP de grado 3, son necesarias mayores ganancias de peso para obtener una moderada reducción del riesgo.

¿Existe un nivel de corte donde una ganancia insu- 
ficiente de peso determine un mayor riesgo de ROP severa susceptible de tratamiento? Para Löfqvist (42) este valor quedaría con ganancias de peso inferiores a 6,5-7,6 gr/día y para Filho (51) este valor quedaría en ganancias de peso inferiores a 7,28 gr/día. En nuestros resultados el límite queda acotado por una ganancia de peso inferior a $7 \mathrm{gr} /$ día; estos prematuros tienen un riesgo de presentar enfermedad plus 14 veces superior al de los prematuros con ganancias de peso $\geq 7 \mathrm{gr} /$ día.

Trabajos recientes y crecientes marcan la importancia de la pobre ganancia de peso postnatal como factor predictivo de la ROP severa susceptible de tratamiento (47-49,51). El presente trabajo, con una elevada razón de verosimilitud, confirma la importancia de una buena ganancia de peso posnatal para reducir el riesgo de enfermedad plus y propone una suficiente ganancia de peso como tratamiento. Reducir el número de días postnatales que el niño pierde peso (fase catabólica), y conseguir una ganancia de peso de $\geq 7$ gr/día, en las 4-6 primeras semanas de vida, deberán ser objetivos prioritarios en el tratamiento de la ROP. Una buena ganancia de peso postnatal es una estrategia efectiva contra la ceguera evitable de la ROP.

\section{BIBLIOGRAFÍA}

1. Hardy RJ, Palmer EA, Dobson V, Summers CG, Phelps DL, Quinn GE, et al. Risk analysis of prethreshold retinopathy of prematurity. Arch Ophthalmol 2003; 121: 16971701 .

2. Early Treatment For Retinopathy Of Prematurity Cooperative Group. Revised indications for the treatment of retinopathy of prematurity: results of the early treatment for retinopathy of prematurity randomized trial. Arch Ophthalmol 2003; 121: 1684-1694.

3. Good WV, Early Treatment for Retinopathy of Prematurity Cooperative Group. Final results of the early treatment for retinopathy of prematurity (ETROP) randomized trial. Trans Am Ophthalmol Soc 2004; 102: 233-250.

4. Multicenter trial of Cryotherapy for retinopathy of prematurity. Preliminary results. Cryotheraphy for retinopathy of prematurity Cooperative Group. Arch Ophthalmol 1988; 106: 471-479.

5. Supplemental Therapeutic Oxygen for Prethreshold Retinopathy Of Prematurity (STOP-ROP), a randomized, controlled trial. I: primary outcomes. Pediatrics 2000; 105: 295-310.

6. International Committee for the Classification of Retinopathy of Prematurity. The International Classification of Retinopathy of Prematurity revisited. Arch Ophthalmol 2005; 123: 991-999.

7. Gelman R, Martinez-Perez ME, Vanderveen DK, Mosko- witz A, Fulton AB. Diagnosis of plus disease in retinopathy of prematurity using retinal image multiScale Analysis. Invest Ophthalmol Vis Sci 2005; 46: 4734-4738.

8. Wallace DK, Freedman SF, Zao Z, Jung SH. Accuracy of $R O P$ tool vs individual examiners in assessing retinal vascular tortuosity. Arch Ophthalmol 2007; 125: 15231530.

9. Funnell CL, Dabbs TR. Assisted conception and retinopathy of prematurity: 8 year follow-up study. Eye 2007; 21: 383-386.

10. Potter JW. El dibujo del fondo. In: Potter JW, Semes LP, Cavallerano AA, Garston MJ. Oftalmoscopia binocular indirecta. Barcelona: JIMS, 1991; 69-79.

11. Rodríguez-Hurtado FJ, Cañizares JM. Despistaje de la retinopatía del prematuro. Nuestra experiencia sobre los límites de peso al nacer, edad gestacional y otros factores de riesgo. Arch Soc Esp Oftalmol 2006; 81: 275-279.

12. Middleton WD, Kurtz AB, Hertzberg BS. Gestación múltiple. In: Middleton WD, Kurtz AB, Hertzberg BS. Ecografía. Madrid: Marban. 2005; 515-529.

13. Strauss A, Paek BW, Genzel-Boroviczény O, Schulze A, Janssen U, Hepp H. Multifetal gestation-maternal and perinatal outcome of 112 pregnancies. Fetal Diagn Ther 2002; 17: 209-217.

14. Luke B, Brown MB, Hediger ML, Misiunas RB, Anderson E. Perinatal and early childhood outcomes of twins versus triplets. Twin Res Hum Genet 2006; 9: 81-88.

15. Landmann E, Misselwitz B, Steiss JO, Gortner L. Mortality an morbidity of neonates born at $<26$ weeks of gestation (1998-2003). A population-based study. J Perinat Med 2008; 36:168-174.

16. Friling $R$, Axer-Siegel $R$, Hersocovici $Z$, Weinberger D, Sirota L, Snir M. Retinopathy of prematurity in assisted versus natural conception and singleton versus multiple births. Ophthalmology 2007; 114: 321-324.

17. Darlow BA, Hutchinson JL, Henderson-Smart DJ, Donoghue DA, Simpson JM, Evans NJ, et al. Prenatal risk factors for severe retinopathy of prematurity among very preterm infants of the Australian an New Zealand Neonatal Network. Pediatrics 2005; 115: 990-996.

18. Montañez FJ, Olea JL. Once años de experiencia en el manejo de la retinopatía del prematuro en las islas Baleares. Arch Soc Esp Oftalmol 2005; 80:713-718.

19. Jalali S, Madhavi C, Reddy GP, Nutheti R. Pilot study on in vivo evaluation of retinal vascular maturity in newborn infants in the context of retinopathy of prematurity. Am J Ophthalmol 2006; 142: 181-183.

20. Chye JK, Lim CT, Leonq HL, Wonq PK. Retinopathy of prematurity in very low birth weight infants. Ann Acad Med Singapore 1999; 28: 193-198.

21. Flynn JT. Acute proliferative retrolental fibroplasia: multivariate risk analysis. Trans Am Ophthalmol Soc 1983; 81: 549-591.

22. Müller H, Weiss C, Kuntz, S, Akkoyun I, Schaible T. Are there predictors for proliferative retinopathy of prematurity and is supplemental oxygen a useful conservative treatment option? Klin Padiatr 2005; 217: 53-60.

23. Shah VA, Yeo CL, Ling YL, Ho LY. Incidence, risk factors of retinopathy of prematurity among very low birth weight infants in Singapore. Ann Acad Med Singapore 2005; 34: 169-178.

24. Hernández M, Orduña C, Bosch V, Salinas R; Alcaraz JL, 
Marín JM. Retinopatía del prematuro en la región de Murcia (España). Incidencia y gravedad. Arch Soc Esp Oftalmol 2008; 83: 423-428.

25. Peralta-Calvo J, Abelairas-Gómez J, Fernández-Guardiola JM, Sánchez-Jacob E. Retinopatía del prematuro $(R P)$ I. Definición y clasificación. Teorías etiológicas, profilaxis y tratamientos médicos. In: Fonseca-Domingo A. Actualización en cirugía Oftálmica pediátrica. Madrid: MacLine SL. 2000; 157-166.

26. Padilla-Vinuesa MC. Alto riesgo obstétrico. In: RuizExtremera A. Niños de riesgo. Programas de atención temprana. Madrid: Norma-Capitel. 2003: 65-74.

27. Fortes Filho JB, Dill JC, Ishizaki A, Aguiar WW, Silveira $R C$, Procianoy RS. Score for neonatal acute physiology and perinatal extension II as a predictor of retinopathy of prematurity: Study in 304 very-low-birth-weight preterm infants. Ophthalmologica 2009; 223: 177-182.

28. Hagadorn JI, Richardson DK, Schmid CH, Cole CH. Cumulative illness severity and progression from moderate to severe retinopathy of prematurity. J Perinatol 2007; 27: 502-509.

29. Drevenstedt GL, Crimmins EM, Vasunilashorn S, Finch $C E$. The rise and fall of excess male infant mortality. Proc Natl Acad Sci USA 2008; 105: 5016-5021.

30. Huo DM, Dong FT, Dai RP, Chu J, Yu WH. Prevalence and risk factors of retinopathy of prematurity. Zhongguo $Y i$ Хие Ke Хиe Yuan Хие Bao 2006; 28: 567-569.

31. Bonotto LB, Moreira AT, Carvalho DS. Prevalence of retinopathy of prematurity in premature babies examined during the period 1992-1999, Joinville (SC): evaluation of associated risks-screening). Arq Bras Oftalmol 2007; 70: 55-61.

32. Ryan S. Nutrition in neonatal chronic lung disease. Eur J Pediatr 1998; 157 S1: 19-22.

33. Larroque B, Ancel PY, Marret S, Marchand L, André M, Arnaud $C$, et al. Neurodevelopmental disabilities and special care of 5-year-old children born before 33 weeks of gestation (the EPIPAGE study): a longitudinal cohort study. Lancet 2008; 371: 813-820.

34. Spear ML, Hassink SG, Leef K, O'Connor DM, Kirwin $S M$, Locke R, et al. Immaturity or starvation? Longitudinal study of leptin levels in premature infants. Biol Neonate 2001; 80: 35-40.

35. Lo HC, Tsao LY, Hsu WY, Chen HN, Yu WK, Chi CY. Relation of cord serum levels of growth hormone, insuline-like growth factors, insuline-like growth factors binding proteins, leptin, and interleukin-6 with birth weight, birth length, and head circumference in term and preterm neonates. Nutrition 2002; 18: 604-608.

36 Nakago S, Ueda Y, Takeuchi K, Maruo T. Implication of maternal nitrogen balance in the regulation of circulating levels of insulin-like growth factor-I in human pregnancy. Endocr J 2002; 49: 299-305.

37. Chen J, Smith LE. Retinopathy of prematurity. Angiogenesis. 2007; 10: 133-140.

38. Hellstrom A, Perruzzi C, Ju M, Engström E, Hard A. Liu $J L$, et al. Low IGF-I suppresses VEGF-survival signaling in retinal endothelial cells: direct correlation with clinical retinopathy of prematurity. Proc Natl Acad Sci USA 2001; 98: 5804-5808.

39. Engström E, Niklasson A, Wikland KA, Ewald U, Hellström A. The role of maternal factors, postnatal nutrition, weight gain, and gender in regulation of serum IGF-1 among preterm infants. Pediatr Res 2005; 57: 605-610.

40. Yeung MY. Somatotropic axis derangement as an underlying factor in the genesis of retinopathy of prematurity. Acta Paediatr 2006; 95: 1334-1340.

41. Price WA, Lee E, Maynor A, Stiles AD, Clemmons DR. Relation between serum insulinlike growth factor-1, insulinlike growth factor binding protein-2, and insulinlike growth factor binding protein-3 and nutritional intake in premature infants with bronchopulmonary dysplasia. $J$ Pediatr Gastroenterol Nutr 2001; 32: 542-549.

42. Löfqvist CH, Engström E, Sigurdsson J, Hard AL, Niklasson A, Ewald U, et al. Postnatal head growth deficit among premature infants parallels retinopathy of prematurity and insulin-like growth factor-1 deficit. Pediatrics 2006; 117: 1930-1938.

43. Villegas-Becerril E, González-Fernández R, Perula-Torres $L$, Gallardo-Galera JM. IGF-I, VEGF y bFGF, como factores pronósticos de la aparición de la retinopatía de la prematuridad. Arch Soc Esp Oftalmol 2006; 81: 641-646.

44. Smith LE. IGF-1 and retinopathy of prematurity in the preterm infant. Biol neonate 2005; 88: 237-244.

45. Hughes $S$, Gardiner T, Baxter L, Chan-Ling T. Changes in pericytes and smooth muscle cells in the kitten model of retinopathy of prematurity: implications for plus disease. Invest Ophthalmol Vis Sci 2007; 48: 1368-1379.

46. Wallace DK, Kylstra JA, Phillips SJ, Hall JG. Poor postnatal weight gain: a risk factor for severe retinopathy of prematurity. JAAPOS 2000; 4: 343-347.

47. Löfqvist C, Andersson E, Sigurdsson J, Engström E, Hard A. Niklasson A, et al. Longitudinal postnatal weight and insulin-like growth factor I measurements in the prediction of retinopathy of prematurity. Arch Ophthalmol 2006; 124: 1711-1718.

48. Hellström A, Hard AL, Engström E, Niklasson A, Andersson E, Smith L, et al. Early weight gain predicts retinopathy in preterm infants: new, simple, efficient approach to screening. Pediatrics 2009; 123: e638-e645.

49. Dhaliwal CA, Fleck BW, Wright E, Graham C, McIntosh $N$. Retinopathy of prematurity in small-for-gestational age infants compared to appropriate-for- gestational age infants. Arch Dis Child Fetal Neonatal Ed 2008; 0: $143552 v 1$.

50. Loos RJ, Derom C, Derom R, Vlientick R. Determinants of birthweight and intrauterine growth in liveborn twins. Paediatr Perinat Epidemiol 2005; S1: 15-22.

51. Filho JB, Bonomo PP, Maia M, Procianoy RS. Weight gain measured at 6 weeks after birth as a predictor for severe retinopathy of prematurity: study with 317 very low birth weight preterm babies. Graefes Arch Clin Exp Ophthalmol 2008; DOI 10.1007/s00417-008-1012-3. 